\title{
Microbleeds after Carotid Artery Stenting: Small Embolism May Induce Cerebral Microbleeds
}

\author{
Ai Ogawa Ito ${ }^{a}$ Akihiro Shindo ${ }^{a}$ Yuichiro li ${ }^{a}$ Keita Matsuura ${ }^{a}$ \\ Ken-ichi Tabei $^{\text {b }}$ Masayuki Maedac $^{c}$ Maki Umino ${ }^{d}$ Yume Suzuki $^{\mathrm{e}}$ \\ Masato Shiba ${ }^{e}$ Naoki Toma ${ }^{e}$ Hidenori Suzuki ${ }^{~}$ Hidekazu Tomimoto $^{a}$ \\ aDepartment of Neurology, Mie University Graduate School of Medicine, Tsu, Japan; \\ ${ }^{b}$ Department of Dementia Prevention and Therapeutics, Mie University Graduate School \\ of Medicine, Tsu, Japan; ' Department of Advanced Diagnostic Imaging, Mie University \\ Graduate School of Medicine, Tsu, Japan; d Department of Radiology, Mie University \\ Graduate School of Medicine, Tsu, Japan; e Department of Neurosurgery, Mie University \\ Graduate School of Medicine, Tsu, Japan
}

\section{Keywords}

Microbleed $\cdot$ Cerebral infarction $\cdot$ Embolism $\cdot$ Carotid artery $\cdot$ Stent

\begin{abstract}
Background: Since the advent of magnetic resonance imaging technology, cerebral microbleeds can be diagnosed in vivo. However, the underlying mechanism of cerebral microbleed formation is not fully understood. Objectives: This study aimed to identify the factors associated with cerebral microbleeds after carotid artery stenting (CAS). Method: We retrospectively examined 125 patients who underwent CAS for carotid stenosis. Cerebral microbleeds were investigated using T2*-weighted gradient-echo (GRE) imaging before and after CAS. We analyzed the possible association of new microbleeds with the following risk factors: the number of baseline microbleeds and ischemic cerebral lesions, the occurrence of cerebral hyperperfusion syndrome, and new ischemic cerebral lesions after CAS. Results: Baseline cerebral microbleeds were detected in 53 patients (42.4\%). New cerebral microbleeds after CAS were observed in 13 of 125 patients (10.4\%) and were exclusively associated with new ischemic lesions but not with other risk factors. No patient showed a merged image of a new cerebral microbleed on GRE imaging or a new ischemic lesion on diffusion-weighted imaging. Lobar and deep microbleeds were noted in 12/13 (92.3\%) and 1 patient (7.7\%), respectively. Of 12 patients with new microbleeds, 10 (76.9\%) and 2 (15.4\%) had a new microbleed in the ipsilat-
\end{abstract}


eral and contralateral hemispheres, respectively. Conclusions: We found that new cerebral microbleeds developed after CAS and that these might be associated with new ischemic lesions, mostly in the territory of the treated carotid artery. We speculate that these microbleeds result from the deoxygenation of hemoglobin in the embolus or, alternatively, small hemorrhagic transformation of ischemic lesions.

\section{Introduction}

Cerebral microbleeds are small perivascular accumulations of hemosiderin-containing macrophages as a corollary of extravasated erythrocytes from cerebral small vessels on histopathologic examination; they appear as small, hypointense, round lesions with a diameter of 5-10 mm on T2*-weighted gradient-echo (GRE) imaging or susceptibility-weighted magnetic resonance imaging (MRI) sequences [1-3]. Histopathologic analyses of small vessels have identified 2 types of vascular pathologic changes: hypertensive vasculopathy and cerebral amyloid angiopathy (CAA). These 2 disorders are characterized by different patterns of microbleed distribution. Hypertensive vasculopathy is typically associated with deep microbleeds in the basal ganglia, thalamus, brainstem, and cerebellum, whereas advanced CAA is associated with lobar microbleeds [3]. Damage to the blood-brain barrier and neurovascular unit may lead to blood leakage and the deposition of hemosiderin [4]. These cerebral microbleeds are classified as primary and secondary microbleeds according to their mechanisms. Primary microbleeds can be caused by erythrocyte extravasation due to interruption of the vascular integrity, whereas secondary microbleeds may be caused by the hemorrhagic transformation of infarction and microinfarction [5].

Cerebral ischemic lesions after cerebral angiography and neurointerventional procedures have been recognized [6], and cerebral embolic lesions after carotid artery stenting (CAS) have been detected on diffusion-weighted MRI in approximately $50-70 \%$ of patients with protection devices [7, 8]. Interestingly, cerebral microbleeds are also found after CAS [9], but the underlying mechanism of cerebral microbleed formation after CAS remains unclear. This study aimed to retrospectively assess the incidence of cerebral microbleeds and identify the possible factors associated with their occurrence after CAS, in order to elucidate the underlying mechanism of their formation.

\section{Materials and Methods}

\section{Patient Involvement}

We performed 176 CAS procedures for symptomatic or asymptomatic carotid stenosis in our hospital from October 2007 to April 2017. Patients were eligible for CAS if they had symptomatic carotid artery stenosis $\geq 50 \%$ or asymptomatic stenosis $\geq 80 \%$, as assessed using digital subtraction angiography, which was performed by following the description in the North American Symptomatic Carotid Endarterectomy Trial [10]. Carotid artery stenosis was considered symptomatic if the patient had a history of ipsilateral ischemic events attributed to the affected carotid artery within the 120 days before CAS, and asymptomatic if no ischemic event had occurred during this period. We included patients who underwent (1) CAS for internal carotid artery stenosis and (2) MRI, including diffusion-weighted imaging (DWI) and T2*-weighted GRE imaging before and the day after CAS. 
Table 1. Clinical, laboratory, and imaging characteristics of patients with or without new cerebral microbleeds

\begin{tabular}{|c|c|c|c|}
\hline & $\begin{array}{l}\text { P-group } \\
(n=13)\end{array}$ & $\begin{array}{l}\text { N-group } \\
(n=112)\end{array}$ & $p$ value \\
\hline Age, years (mean $\pm S D)$ & $72.2 \pm 5.9$ & $72.2 \pm 7.2$ & 0.99 \\
\hline Male gender & $12(92.3)$ & $101(90.2)$ & 1.00 \\
\hline \multicolumn{4}{|l|}{ Risk factors } \\
\hline Hypertension & $12(92.3)$ & $91(81.2)$ & 0.46 \\
\hline Diabetes & $5(38.5)$ & $36(32.1)$ & 0.76 \\
\hline Dyslipidemia & $10(76.9)$ & $90(80.4)$ & 0.73 \\
\hline Atrial fibrillation & $1(7.7)$ & $4(3.6)$ & 0.43 \\
\hline Smoking & $12(92.3)$ & $86(76.8)$ & 0.30 \\
\hline Alcohol & $5(38.5)$ & $55(49.1)$ & 0.56 \\
\hline Carotid stenosis ${ }^{1}, \%($ mean \pm SD) & $79.7 \pm 10.7$ & $78.7 \pm 13.0$ & 0.78 \\
\hline \multicolumn{4}{|l|}{ MRI findings before CAS } \\
\hline CMBs & $5(38.5)$ & $48(42.9)$ & 1.00 \\
\hline Pure lobar CMBs & $1(7.7)$ & $18(16.1)$ & 0.69 \\
\hline Pure deep CMBs & $3(23.1)$ & $17(15.2)$ & 0.44 \\
\hline Mixed lobar and deep CMBs & $1(7.7)$ & $13(11.6)$ & 1.00 \\
\hline Median number of deep MBs (range) & $0(0-2)$ & $0(0-18)$ & 0.56 \\
\hline Median number of lobar MBs (range) & $0(0-1)$ & $0(0-7)$ & 0.30 \\
\hline cSS on GRE imaging & $3(23.1)$ & $3(2.7)$ & 0.02 \\
\hline Infarction on DWI & $6(46.2)$ & $47(42.0)$ & 0.78 \\
\hline \multicolumn{4}{|l|}{ MRI findings after CAS } \\
\hline Embolic infarction on DWI & $11(84.6)$ & $51(45.5)$ & 0.01 \\
\hline Statin use & $11(84.6)$ & $90(80.4)$ & 1.00 \\
\hline CHS after CAS & $0(0)$ & $11(9.8)$ & 0.60 \\
\hline
\end{tabular}

Values represent $n(\%)$ unless otherwise indicated. CMBs, cerebral microbleeds; P-group, patients positive for new CMBs; N-group, patients negative for new CMBs; CAS, carotid artery stenting; GRE, T2*-weighted gradient-echo; DWI, diffusion-weighted imaging; cSS, cortical superficial siderosis; CHS, cerebral hyperperfusion syndrome.

${ }^{1}$ According to the NASCET (North American Symptomatic Carotid Endarterectomy Trial) criteria.

The protocols of the CAS procedure, MRI, and single-photon emission computed tomography (SPECT) are outlined in the online supplementary materials (for all online suppl. material, see www.karger.com/doi/10.1159/000500112).

\section{Clinical Assessment}

We obtained clinical, laboratory, and imaging data upon admission and after CAS. Patients' characteristics including age, sex, hypertension, diabetes mellitus, dyslipidemia, atrial fibrillation, smoking, alcohol, antithrombotic therapy (e.g., dual antiplatelet therapy and in combination with an anticoagulant), and statin use were examined. Moreover, we detected the dominance of both anterior cerebral arteries on MR angiography and cerebral angiography.

\section{Statistical Analysis}

Continuous variables are presented as mean \pm standard deviation or median (range), whereas categorical variables are reported as $n(\%)$. For the univariate analysis, we used Student's $t$ test to analyze continuous data and the $\chi^{2}$ test or Fisher's exact test to analyze cate- 


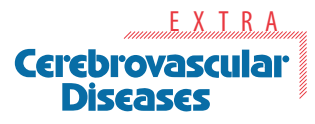

Table 2. Generalized linear model analysis of the development of new CMBs

\begin{tabular}{l|l}
\hline Cerebrovasc Dis Extra 2019;9:57-65 \\
\hline DOI: 10.1159/000500112 & $\begin{array}{l}\text { ○ 2019 The Author(s). Published by S. Karger AG, Basel } \\
\text { www.karger.com/cee }\end{array}$ \\
\hline
\end{tabular}

Ogawa Ito et al.: Microbleeds after CAS

\begin{tabular}{lll}
\hline & OR $(95 \% \mathrm{CI})$ & $p$ value \\
\hline CMBs before CAS & $0.49(0.12-2.02)$ & 0.33 \\
New ischemic lesions after CAS & $6.54(1.32-32.3)$ & 0.02 \\
cSS & $12.9(1.61-102.4)$ & 0.02 \\
HTN & $2.56(0.32-22.1)$ & 0.39 \\
\hline
\end{tabular}

CAS, carotid artery stenting; CMBs, cerebral microbleeds; cSS, cortical superficial siderosis; HTN, hypertension; OR, odds ratio; $\mathrm{CI}$, confidence interval.

Table 3. Demographics of patients with new cerebral microbleeds

\begin{tabular}{|c|c|c|c|c|c|c|c|c|c|}
\hline \multirow{2}{*}{$\begin{array}{l}\text { Case } \\
\text { No. }\end{array}$} & \multirow{2}{*}{$\begin{array}{l}\text { Age, } \\
\text { years }\end{array}$} & \multirow{2}{*}{$\begin{array}{l}\text { Side, } \\
\text { R/L }\end{array}$} & \multirow[t]{2}{*}{ CHS } & \multicolumn{2}{|c|}{ New ischemic lesions } & \multicolumn{2}{|c|}{ New cerebral microbleeds } & \multirow{2}{*}{$\begin{array}{l}\text { Deep or } \\
\text { lobar }\end{array}$} & \multirow{2}{*}{$\begin{array}{l}\text { Hemisphere } \\
\text { to CAS }\end{array}$} \\
\hline & & & & $n$ & location & $\bar{n}$ & location & & \\
\hline 1 & 62 & $\mathrm{R}$ & No & 4 & $\mathrm{RF}, \mathrm{RP}, \mathrm{LF}$ & 1 & LF & lobar & contralateral \\
\hline 2 & 78 & $\mathrm{~L}$ & No & 3 & LF, LT, LO & 1 & LF & lobar & ipsilateral \\
\hline 3 & 74 & $\mathrm{~L}$ & No & 4 & $\mathrm{RF}, \mathrm{LF}$ & 1 & LF & lobar & ipsilateral \\
\hline 4 & 74 & $\mathrm{R}$ & No & 1 & $\mathrm{RP}$ & 1 & $\mathrm{RP}$ & lobar & ipsilateral \\
\hline 5 & 79 & $\mathrm{~L}$ & No & 4 & LF, LT & 1 & LF & lobar & ipsilateral \\
\hline 6 & 73 & $\mathrm{R}$ & No & 0 & none & 1 & $\mathrm{RF}$ & lobar & ipsilateral \\
\hline 7 & 80 & $\mathrm{R}$ & No & 2 & RT, RP & 1 & $\mathrm{RF}$ & lobar & ipsilateral \\
\hline 8 & 73 & $\mathrm{~L}$ & No & 3 & LO & 1 & LO & lobar & ipsilateral \\
\hline 9 & 75 & $\mathrm{R}$ & No & 2 & RF, RP & 1 & $\mathrm{RF}$ & lobar & ipsilateral \\
\hline 10 & 68 & $\mathrm{R}$ & No & 3 & $\mathrm{RF}, \mathrm{RT}$ & 1 & Ro & lobar & ipsilateral \\
\hline 11 & 75 & $\mathrm{~L}$ & No & 4 & RP, LP, LO & 1 & $\mathrm{RF}$ & lobar & contralateral \\
\hline 12 & 72 & $\mathrm{~L}$ & No & 0 & none & 1 & LT & lobar & ipsilateral \\
\hline 13 & 67 & $\mathrm{~L}$ & No & 3 & RF, LF, LT & 1 & RM & deep & ipsilateral \\
\hline
\end{tabular}

M, male, F, female, R, right; L, left; F, frontal lobe; T, temporal lobe; P, parietal lobe; O, occipital lobe; M, midbrain; CAS, carotid artery stenting; CHS, cerebral hyperperfusion syndrome.

gorical data. A generalized linear model was used to examine the relationship between cerebral microbleeds before CAS, a new ischemic infarction after CAS, cortical superficial siderosis (cSS) before CAS, and new cerebral microbleeds after CAS. A $p$ value $<0.05$ was considered statistically significant. Statistical analyses were performed using SPSS v24 (IBM, Armonk, NY, USA).

\section{Results}

\section{Patient Characteristics}

During the study period, 176 patients underwent CAS at our hospital; 51 patients who did not undergo T2*-weighted GRE imaging before or after CAS were excluded. Thus, 125 (113 males and 12 females) were included in the final analysis (online suppl. Fig. S1). The mean age at the time of CAS was $72.2 \pm 7.0$ (range 50-87) years.

Clinical, laboratory, and imaging characteristics of patients with (P-group) or without (N-group) new postprocedural cerebral microbleeds are shown in Tables 1, 2 and in online supplementary Table S1. The demographics of the P-group are summarized in Table 3. Figure 1 shows representative MRI of the P-group. SPECT and angiographic findings in the P-group are shown in online supplementary Figure S2. 


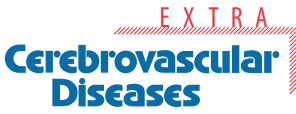

\begin{tabular}{l|l}
\hline Cerebrovasc Dis Extra 2019;9:57-65 \\
\hline DOI: 10.1159/000500112 & $\begin{array}{l}\text { ○ 2019 The Author(s). Published by S. Karger AG, Basel } \\
\text { www.karger.com/cee }\end{array}$ \\
\hline
\end{tabular}

Ogawa Ito et al.: Microbleeds after CAS
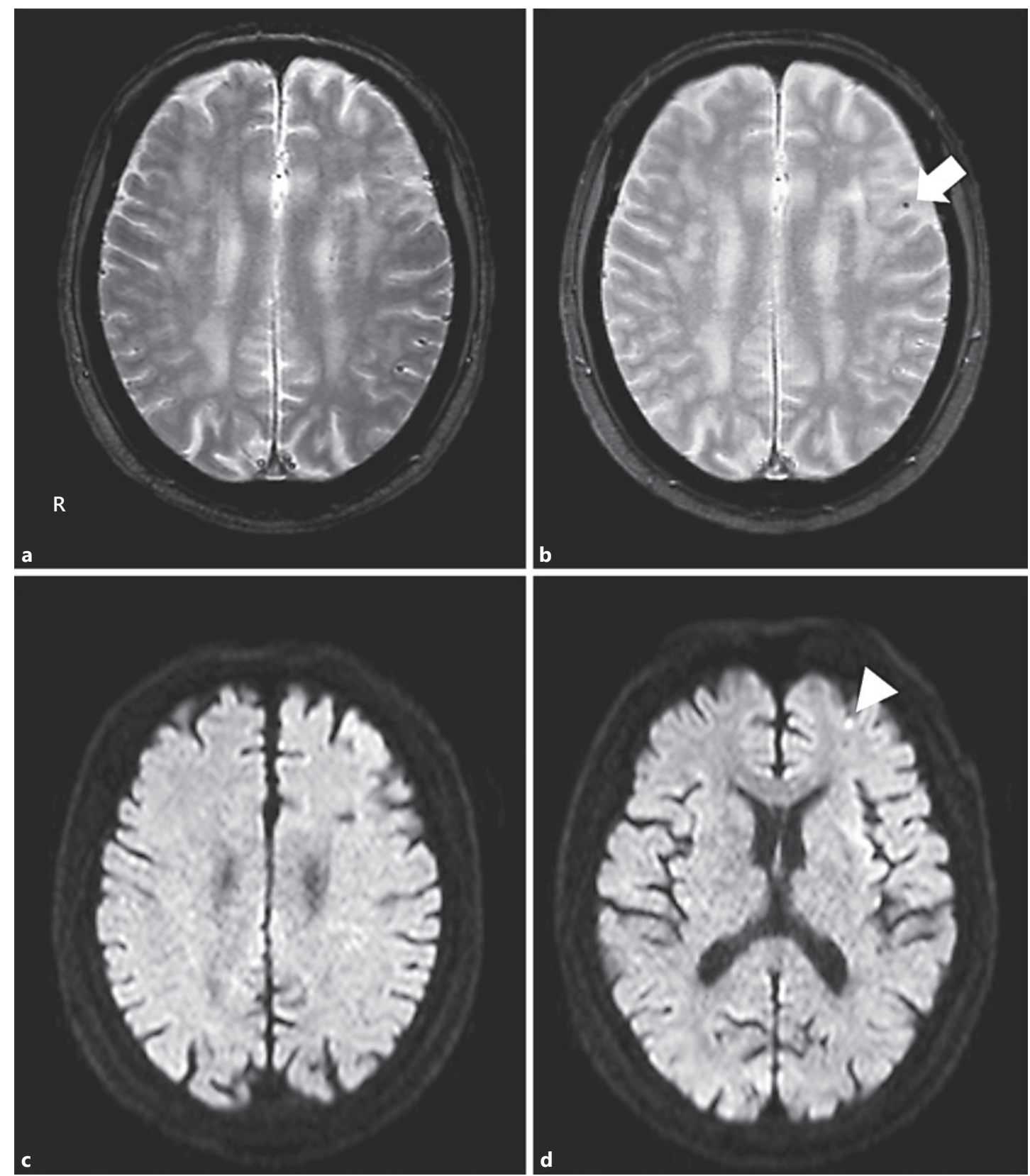

Fig. 1. Representative magnetic resonance images of a new cerebral microbleed after carotid artery stenting (CAS). a T2*-weighted gradient-echo (GRE) imaging 1 day before CAS did not detect a microbleed in a 74-year-old man (case 3 in Table 2). b GRE imaging 1 day after CAS of the left internal carotid artery showed the development of a new cerebral microbleed (arrow) in the left frontal lobe. c Diffusion-weighted imaging (DWI) after CAS showed no ischemic lesion around the microbleed. $\mathbf{d}$ DWI indicated a new ischemic lesion in the left frontal lobe (arrowhead).

\section{MRI and SPECT Findings}

Baseline cerebral microbleeds were detected in 53 patients (42.4\%). Of these, 19 (35.8\%), $20(37.7 \%)$, and $14(26.4 \%)$ had pure lobar, pure deep, and mixed lobar and deep microbleeds, respectively. New postprocedural cerebral microbleeds were detected in only 13 patients (10.4\%). Baseline ischemic lesions before CAS were noted in 53 patients $(42.4 \%)$. 
New postprocedural ischemic lesions were detected in 62 patients (49.6\%). Notably, no patient showed a merged image of a new cerebral microbleed on GRE imaging or a new ischemic lesion on DWI; cSS was detected in 6 patients (4.8\%) on baseline GRE imaging.

Cerebral hyperperfusion syndrome (CHS) was detected in 11 patients after CAS based on the results of SPECT. All 11 patients belonged to the N-group.

\section{Factors Associated with New Cerebral Microbleeds}

New DWI-positive lesions after CAS were detected in 51 patients in the N-group (45.5\%) and 11 in the P-group (84.6\%). In the univariate analysis, cerebral microbleeds after CAS were significantly associated with the presence of new postprocedural cerebral ischemic lesions due to artery-to-artery embolism $(p=0.008)$. The frequency of cSS before CAS was higher in the P-group than in the N-group $(p=0.015)$. No significant differences in hypertension, diabetes mellitus, hyperlipidemia, atrial fibrillation, smoking, alcohol, CHS, antithrombotic therapy, or the presence of cerebral microbleeds and DWI-positive ischemic lesions before CAS were observed in the 2 groups (Table 1; online suppl. Table 1). In the multivariate analysis, the presence of a new embolic infarction after CAS and cSS before CAS was an independent predictor of the development of new cerebral microbleeds after CAS. Hypertension and the presence of cerebral microbleeds before CAS were not associated with the appearance of new cerebral microbleeds (Table 2).

\section{Demographics of Patients with New Postprocedural Cerebral Microbleeds}

Of 13 patients with new cerebral microbleeds, 12 (92.3\%) and 1 (7.7\%) had lobar and deep microbleeds, respectively. New lobar microbleeds in the ipsilateral and contralateral hemispheres were detected in 10 (76.9\%) and 2 (15.4\%) patients, respectively. Two patients showed cerebral microbleeds in the frontal lobe of the contralateral hemisphere after CAS and had a bilateral supply from the anterior cerebral arteries arising from the ipsilateral internal carotid artery with severe stenosis, indicating the possibility of contralateral embolization. All newly detected lobar microbleeds were found in the territory of the treated carotid artery (Table 3).

\section{Discussion}

In this study, we showed a novel finding of cerebral microbleeds that might have been caused by artery-to-artery embolism after CAS, because all new lobar microbleeds after CAS were detected in the territory of the treated carotid artery and were spatially associated with a cerebral microembolism. This may indicate that small, hypointense lesions noted on T2*weighted GRE imaging might be caused by hemorrhagic microinfarction. Cerebral microbleeds are associated with many risk factors, such as age, male sex, hypertension, and low total cholesterol [11].

Fisher [5] reported that cerebral microbleeds are classified as primary and secondary microbleeds based on the underlying mechanisms. Primary cerebral microbleeds represent the perivascular accumulation of hemosiderin-laden macrophages as a corollary of extravasated erythrocytes from cerebral small vessels on histopathologic examination [1]. Secondary microbleeds may be caused by another mechanism including hemorrhagic infarction and microinfarction [5]. Pathologic analysis has shown that a substantial proportion of cerebral microhemorrhages are associated with microinfarcts [12]. Ischemia may cause the extracellular transfer of iron stored in oligodendrocytes $[5,12]$.

Although the embolic mechanism may induce cerebral microbleeds, no patient showed a completely merged image of a new cerebral microbleed on GRE imaging or a new ischemic 
lesion on DWI. If all new cerebral microbleeds were due to the hemorrhagic transformation of a new microembolism, some of them would have shown as a merged image of microbleeds and microinfarcts on MRI at the acute stage. Therefore, the absence of a merged image may indicate that cerebral microbleeds at the acute stage correspond to lodged emboli per se, or, alternatively, extravasated emboli, which is termed angiophagy $[14,15]$. Angiophagy represents the phenomenon of the phagocytosis of microemboli by vascular endothelial cells and extrusion into the perivascular space $[5,13]$. Angiophagy is a mechanism of microvascular recanalization that occurs in the terminal capillaries or arterioles 1-6 days after embolization $[14,15]$. Irrespective of their location inside or outside of the microvessels, microemboli can exhibit a paramagnetic effect via the deoxygenation of hemoglobin within a few hours at the earliest [16]. Moreover, imaging-histopathologic correlation studies have shown that cerebral microbleeds detected by blood-sensitive sequences on MRI can represent acute and chronic microhemorrhages $[17,18]$. Histopathologic analysis showed a microbleed that corresponded to intact erythrocytes, indicating an acute hemorrhage [17]. Another case report described a microbleed at the subacute microhemorrhage stage on $\mathrm{T} 2 *$-weighted GRE imaging [18]. In our study, microbleeds shown on GRE imaging the day after CAS might have reflected erythrocytes containing acute deoxyhemoglobin but not hemosiderincontaining macrophages.

Previous studies have shown that microemboli in animal models enter the penetrating artery and can cause lacunar and microinfarction. In a monkey model, cortical and internal watershed infarcts were observed after the injection of $>2,000$ microspheres per internal carotid artery. Moreover, MRI showed small, round hypointensities which met the criteria for microbleeds associated with ischemic lesions [19]. Moreover, microhemorrhages have been observed in a mouse model of embolic cerebral ischemia [20]. Thus, microembolism may lead to hemorrhagic (micro-)infarction or, alternatively, embolus extravasation at the capillary level which has been seen in animal models after embolization [21].

A previous study showed that cerebral microbleeds developed after CAS in $8.0 \%$ of patients [9]; this is in agreement with the $10.4 \%$ rate of occurrence noted in this study. The previous study suggested that the presence of baseline cerebral microbleeds was a predictor of the appearance of a new one, and hemodynamic changes during CAS might have damaged the small cerebral vessels. In contrast, our study showed that new cerebral microbleeds were associated with new ischemic lesions, mostly in the same vascular territory but not with baseline microbleeds.

In addition, the presence of cSS before CAS tended to be associated with the development of cerebral microbleeds after CAS. It has been suggested that cSS is a neuroimaging biomarker of CAA, with some studies reporting a high prevalence of cSS in CAA cases [2]. Our study may indicate that CAA might pose the risk of developing new microbleeds after CAS.

Our study has several limitations. First, the results of this research may have limited statistical implications because of the small number of cases. Second, our study had a retrospective design with the potential risk of selection bias. Third, although we described cerebral microbleeds and ischemic lesions using MRI, its histopathology remains unknown. Several previous studies reported foreign-body emboli following cerebral angiography, coronary angiography, or the endovascular treatment of intracranial angiography [22-25]. A hydrophilic polymer could be an iatrogenic cause of infarction [22, 25], but this is not likely the cause of artifactual microbleeds; no patient presented with any symptom suggesting delayed allergic reactions after CAS. Although both cerebral microbleeds and microinfarction were observed closely together after CAS, it was difficult to find a new ischemic lesion that transformed into a new microbleed in this study. Further studies are necessary to explore microbleeds induced by small embolisms. 
Our study showed that new cerebral microbleeds developed after CAS, and that this phenomenon might be associated with new ischemic lesions. We speculate that the new microbleeds resulted from angiophagy or small hemorrhagic transformation of embolic infarctions.

\section{Acknowledgement}

We would like to express our gratitude to the patients for their cooperation.

\section{Statement of Ethics}

This study was performed with the approval of the ethics committee of Mie University Hospital and conformed to the tenets of the Declaration of Helsinki. The requirement for obtaining patients' written informed consent for the retrospective review of medical records was waived. Patients involved in this study and their families were granted the right to opt out.

\section{Disclosure Statement}

The authors have no conflicts of interest to declare.

\section{Funding Sources}

This work was supported in part by SPS KAKENHI (grant No. 18K16557).

\section{Author Contributions}

Ai Ogawa Ito, Akihiro Shindo, and Hidekazu Tomimoto were responsible for the study concept and interpretation of data. Ai Ogawa Ito and Akihiro Shindo contributed equally to this paper. Ai Ogawa Ito, Akihiro Shindo, Yuichiro Ii, Ken-ichi Tabei, Maeda Masayuki, Maki Umino, Yume Suzuki, Masato Shiba, Naoki Toma, and Hidenori Suzuki contributed to the acquisition and analysis of data. Finally, all authors participated in the critical revision and approval of the manuscript.

\section{References}

1 Fazekas F, Kleinert R, Roob G, Kleinert G, Kapeller P, Schmidt R, et al. Histopathologic analysis of foci of signal loss on gradient-echo $\mathrm{T} 2 *$-weighted MR images in patients with spontaneous intracerebral hemorrhage: evidence of microangiopathy-related microbleeds. AJNR Am J Neuroradiol. 1999 Apr;20(4):637-42.

2 Charidimou A, Kakar P, Fox Z, Werring DJ. Cerebral microbleeds and recurrent stroke risk: systematic review and meta-analysis of prospective ischemic stroke and transient ischemic attack cohorts. Stroke. 2013 Apr; 44(4):995-1001.

3 Greenberg SM, Vernooij MW, Cordonnier C, Viswanathan A, Al-Shahi Salman R, Warach S, et al; Microbleed Study Group. Cerebral microbleeds: a guide to detection and interpretation. Lancet Neurol. 2009 Feb;8(2): 165-74. 
Ogawa Ito et al.: Microbleeds after CAS

4 Wang Z, Soo YO, Mok VC. Cerebral microbleeds: is antithrombotic therapy safe to administer? Stroke. 2014 Sep;45(9):2811-7.

5 Fisher M. Cerebral microbleeds: where are we now? Neurology. 2014 Oct;83(15):1304-5.

6 Bendszus M, Koltzenburg M, Burger R, Warmuth-Metz M, Hofmann E, Solymosi L. Silent embolism in diagnostic cerebral angiography and neurointerventional procedures: a prospective study. Lancet. 1999 Nov; 354(9190):1594-7.

7 Bijuklic K, Wandler A, Hazizi F, Schofer J. The PROFI study (Prevention of Cerebral Embolization by Proximal Balloon Occlusion Compared to Filter Protection during Carotid Artery Stenting): a prospective randomized trial. J Am Coll Cardiol. 2012 Apr;59(15):1383-9.

8 Tanemura H, Maeda M, Ichikawa N, Miura Y, Umeda Y, Hatazaki S, et al. High-risk plaque for carotid artery stenting evaluated with 3-dimensional T1-weighted gradient echo sequence. Stroke. 2013 Jan;44(1):105-10.

9 Kakumoto K, Matsumoto S, Nakahara I, Watanabe Y, Fukushima Y, Yoshikiyo U, et al. Rapid formation of cerebral microbleeds after carotid artery stenting. Cerebrovasc Dis Extra. 2012 Jan-Apr;2(1):9-16.

10 Barnett HJ, Taylor DW, Haynes RB, Sackett DL, Peerless SJ, Ferguson GG, et al; North American Symptomatic Carotid Endarterectomy Trial Collaborators. Beneficial effect of carotid endarterectomy in symptomatic patients with high-grade carotid stenosis. N Engl J Med. 1991 Aug;325(7):445-53.

11 Romero JR, Preis SR, Beiser A, DeCarli C, Viswanathan A, Martinez-Ramirez S, et al. Risk factors, stroke prevention treatments, and prevalence of cerebral microbleeds in the Framingham Heart Study. Stroke. 2014 May;45(5):1492-4.

12 Tanskanen M, Makela M, Myllykangas L, Rastas S, Sulkava R, Paetau A. Intracerebral hemorrhage in the oldest old: a population-based study (Vantaa 85+). Front Neurol. 2012 Jun;3:103.

13 Grutzendler J, Murikinati S, Hiner B, Ji L, Lam CK, Yoo T, et al. Angiophagy prevents early embolus washout but recanalizes microvessels through embolus extravasation. Sci Transl Med. 2014 Mar;6(226):226ra31.

14 Grutzendler J. Angiophagy: mechanism of microvascular recanalization independent of the fibrinolytic system. Stroke. 2013 Jun;44(6 Suppl 1):S84-6.

15 Lam CK, Yoo T, Hiner B, Liu Z, Grutzendler J. Embolus extravasation is an alternative mechanism for cerebral microvascular recanalization. Nature. 2010 May;465(7297):478-82.

16 Cho KH, Kim JS, Kwon SU, Cho AH, Kang DW. Significance of susceptibility vessel sign on T2*-weighted gradient echo imaging for identification of stroke subtypes. Stroke. 2005 Nov;36(11):2379-83.

17 van Veluw SJ, Biessels GJ, Klijn CJ, Rozemuller AJ. Heterogeneous histopathology of cortical microbleeds in cerebral amyloid angiopathy. Neurology. 2016 Mar;86(9):867-71.

18 Boulouis G, Charidimou A, Van Veluw S, Greenberg SM. Imaging the acute formation of a cortical microbleed in cerebral amyloid angiopathy. JAMA Neurol. 2017 Jan;74(1):120-1.

19 Maki T, Wakita H, Mase M, Itagaki I, Saito N, Ono F, et al. Watershed infarcts in a multiple microembolic model of monkey. Neurosci Lett. 2011 Jul;499(2):80-3.

20 Akai F, Maeda M, Hashimoto S, Taneda M, Takagi H. A new animal model of cerebral infarction: magnetic embolization with carbonyl iron particles. Neurosci Lett. 1995 Jul;194(1-2):139-41.

21 Wakita H, Shindo A, Tomimoto H. What is the aftermath of cerebral microembolism? J Alzheimers Dis Parkinsonism. 2017;7(5):1000375.

22 Mehta RI, Mehta RI, Solis OE, Jahan R, Salamon N, Tobis JM, et al. Hydrophilic polymer emboli: an under-recognized iatrogenic cause of ischemia and infarct. Mod Pathol. 2010 Jul;23(7):921-30.

23 Yasuda R, Maeda M, Umino M, Nakatsuka Y, Umeda Y, Toma N, et al. Suspected metallic embolism following endovascular treatment of intracranial aneurysms. AJNR Am J Neuroradiol. 2016 Sep;37(9):1696-9.

24 Jassal DS, Fast MD, McGinn G. Multifocal brain MRI hypointensities secondary to cardiac catheterization. Neurology. 2000 May;54(10):2023-4.

25 Shapiro M, Ollenschleger MD, Baccin C, Becske T, Spiegel GR, Wang Y, et al. Foreign body emboli following cerebrovascular interventions: clinical, radiographic, and histopathologic features. AJNR Am J Neuroradiol. 2015 Nov;36(11):2121-6. 